\title{
Abundant closed form wave solutions to the Burgers equation, Bogoyavlenskii equation and negative Gardner-KP equation
}

\author{
H. M. Shahadat Ali ${ }^{1}$, C. M. Akher ${ }^{2}$, M. Mamun Miah ${ }^{3}$ and M. Ali Akbar ${ }^{4}$ \\ ${ }^{1}$ Department of Applied Mathematics, Noakhali Science and Technology University, Bangladesh \\ ${ }^{2}$ Department of Mathematics, Bangladesh Army University of Engineering and Technology, Natore, 6431, Bangladesh \\ ${ }^{3}$ Department of Mathematics, Khulna University of Engineering and Technology, Khulna-9203, Bangladesh \\ ${ }^{4}$ Department of Applied Mathematics, University of Rajshahi,Rajshahi-6205, Bangladesh
}

Received: 23 October 2018, Accepted: 29 May 2019

Published online: 13 February 2020.

\begin{abstract}
The (1+1)-dimensional Burgers equation, the (2+1)-dimensional Bogoyavlenskii equation and the $(2+1)$-dimensional negative Gardner-KP equation are important physical model to analyze signal processing system, optical fiber, distinct topological signature in the phase space, nonlinear wave, shock wave, complex solitary wave, shallow water surface wave etc. Therefore, the investigation of closed form traveling wave solutions of these equations play a vital role to reveal the inner mechanism of these physical events. In this article, we examine new closed form traveling wave solutions of the above mentioned nonlinear evolution equations (NLEEs) by using an extended method known as the two variable $\left(G^{\prime} / G, 1 / G\right)$-expansion method, where $G$ satisfies the second order differential equation. In this method, we reduce the NLEEs to nonlinear ordinary differential equations by using the wave coordinate transformation and the traveling wave solutions are expressed in the form of hyperbolic function, trigonometric function and rational function. This method can be considered as the generalization of the well-known $\left(G^{\prime} / G\right)$-expansion method given by Wang et al. and it is shown that the suggested method to be easier and faster by means of a symbolic computation system.
\end{abstract}

Keywords: Two variable $\left(G^{\prime} / G, 1 / G\right)$-expansion method, Burgers equation, Bogoyavlenskii equation, negative Gardner-KP equation, traveling wave solutions.

\section{Introduction}

Nonlinear phenomena exist in all branches of science and engineering fields, such as optical fibers, elastic media, solid state physics, plasma physics, chemical physics, chemical kinematics, biology, atmospheric phenomena, fluid mechanics and so on. The nonlinear physical phenomena can be represented by nonlinear differential equations which are partial or ordinary. Therefore, the exact solutions of these equations provided much physical information and help to understand the mechanism of the existent state. The closed form solutions of these NLEEs help to comprehend the nonlinear physical phenomena. For this reason, to explore closed form wave solutions of NLEEs is very important. In the last few decades, the researchers developed some useful and efficient methods to examine closed form solution to nonlinear equations. Now a days, with the help of computation packages like Mathematica several methods have been proposed, developed and extended, such as the tanh-function expansion method [1], the Jocobi elliptic function expansion method [2, 3], the homogeneous balance method [4, 5], the F-expansion method [6], the sub-ODE method [7], the exp-function expansion method $[8,9,10,11]$, the Backlund and Darboux transformation $[12,13]$, the tanh-sech method $[14,15,16]$, the extended tanh method [17], the sine-cosine method $[18,19,20]$ and the $\left(G^{\prime} / G\right)$-expansion method $[21,22,23,24]$ and so on. In order to better understand a method and to expand the range of applicability it is essential to propose diverse extension of this method. Thus, Akbar et al. [25] derive a generalized and improved $\left(G^{\prime} / G\right)$-expansion method for examining 
nonlinear evolution equations. By using extended $\left(G^{\prime} / G\right)$-expansion method, Zayed and Al-Joudi [26] investigated two remarkable equations. Li et al. [27] first introduced $\left(G^{\prime} / G, 1 / G\right)$-expansion method and successfully investigated the Zakharov equations. After that, many researchers in references [28, 29, 30, 31] used this method and determined closed form solution of the NLEEs. Miah et al. [32] and Ali et al. [33] also studied exact solution of NLEEs by using this eminent method and effectively get useful results. Very recently, Miah et al. [34] applied the $\left(G^{\prime} / G, 1 / G\right)$-expansion method for examining abundant traveling wave solutions to the $(2+1)$-dimensional typical breaking soliton equation and the $(1+1)$-dimensional classical Boussinesq equation. But, the $(1+1)$-dimensional Burgers equation, the $(2+1)$ dimensional Bogoyavlenskii equation and the $(2+1)$-dimensional negative Gardner-KP equations have not been studied by anyone through the $\left(G^{\prime} / G, 1 / G\right)$-expansion method. The objective of this article is to investigate much new and general wave solutions of the above mentioned equations by making use of the two variable $\left(G^{\prime} / G, 1 / G\right)$-expansion method. The $(1+1)$-dimensional Burgers equation $[35,36]$ is

$$
u_{t}+u u_{x}=u_{x x}
$$

This equation is used as a mathematical model in turbulence problem, in the theory of shock wave and in continuous stochastic process. It is also used in vehicle density in high way traffic. It is one of the fundamental PDEs in fluid mechanics. Burgers equation is completely integrable and the solutions of this equation are single and multiple front solution. The (2+1)-dimensional Bogoyavlenskii equation $[37,38]$ is

$$
\begin{aligned}
& 4 u_{t}+u_{x x y}-4 u^{2} u_{y}-4 u_{x} v=0 \\
& u u_{y}=v_{x}
\end{aligned}
$$

In this physical model, the linear superposition of the shock wave solution and the complex solitary wave solution for the physical field is still a solution of the equation of interest, except for a phase-shift. The $(2+1)$-dimensional Negative Gardner-KP equation $[39,40]$ is

$$
\left(u_{t}+6 u u_{x}-6 u^{2} u_{x}+u_{x x x}\right)_{x}+u_{y y}=0
$$

This is the combined KdV-mKdV equation can produces physical models which describe the internal solitary waves in shallow seas, known as negative Gardner-KP equation. It will be positive Gardner-KP equation or negative Gardner-KP equation depending on the sign of the cubic nonlinear term. The rest of this article is organized as follows: In section 2 , we present the explanation of the two variable $\left(G^{\prime} / G, 1 / G\right)$-expansion method. In section 3, some applications of the delineated method are scrutinized. In section 4 , results and discussions are provided and finally, in section 5 , the conclusions are drawn.

\section{Explanation of the two variable $\left(G^{\prime} / G, 1 / G\right)$-expansion method}

In this section, we designate the main steps of the two variable $\left(G^{\prime} / G, 1 / G\right)$-expansion method for finding traveling wave solutions of the denoted NLEEs. Let us consider the second order linear ordinary differential equation (LODE) as follows:

$$
G^{\prime \prime}(\xi)+\lambda G(\xi)=\mu
$$

and associate the relations

$$
\phi=G^{\prime} / G, \psi=1 / G
$$

Thus, from (4) and (5) it can be established

$$
\phi^{\prime}=-\phi^{2}+\mu \psi-\lambda, \quad \psi^{\prime}=-\phi \psi
$$


The solutions of the above equation (4) figure on $\lambda$. Therefore,depending on the sign of $\lambda$, there arises three cases as follows:

Case 1: When $\lambda<0$,the general solution of LODE (4) is as follows:

$$
G(\xi)=A_{1} \sinh (\sqrt{-\lambda} \xi)+A_{2} \cosh (\sqrt{-\lambda} \xi)+\frac{\mu}{\lambda}
$$

where $A_{1}$ and $A_{2}$ are two arbitrary constants. Consequently it can be derived

$$
\psi^{2}=\frac{-\lambda\left(\phi^{2}-2 \mu \psi+\lambda\right)}{\lambda^{2} \sigma+\mu^{2}}
$$

in where $\sigma=A_{1}^{2}-A_{2}^{2}$.

Case 2: When $\lambda>0$, the general solution of LODE (4) is as follows:

$$
G(\xi)=A_{1} \sin (\sqrt{\lambda} \xi)+A_{2} \cos (\sqrt{\lambda} \xi)+\frac{\mu}{\lambda}
$$

and hence

$$
\psi^{2}=\frac{\lambda\left(\phi^{2}-2 \mu \psi+\lambda\right)}{\lambda^{2} \rho-\mu^{2}}
$$

wherein $\rho=A_{1}^{2}+A_{2}^{2}$.

Case 3: When $\lambda=0$, the general solution of LODE (4) is as follows:

$$
G(\xi)=\frac{\mu}{2} \xi^{2}+A_{1} \xi+A_{2}
$$

Thus,

$$
\psi^{2}=\frac{\left(\phi^{2}-2 \mu \psi\right)}{A_{1}^{2}-2 \mu A_{2}}
$$

Let us consider the following nonlinear evolution equation in three independent variables $x, y, t$,

$$
Q\left(u, u_{t}, u_{x}, u_{y}, u_{t t}, u_{x x}, u_{y y}, u_{x y}, \ldots\right)=0
$$

where $Q$ is a polynomial in wave function $u(x, y, t)$ and its partial derivatives. In the following, we give the most important steps of the two variable $\left(G^{\prime} / G, 1 / G\right)$-expansion method which is given in details in Ref. $[27,28,29,30,31,32,33,34]$.

Step 1: Combining the independent variables into one wave variable, we suppose that

$$
u(x, y, t)=u(\xi), \xi=x+y-c t
$$

where $c$ is the celerity of the wave and the traveling wave variable Eq. (14) permits us in reducing Eq. (13) into an ODE for $u(\xi)$ as

$$
R\left(u,-c u^{\prime}, u^{\prime}, u^{\prime}, c^{2} u^{\prime \prime}, u^{\prime \prime}, u^{\prime \prime}, \ldots\right)=0
$$


where $R$ is a polynomial of $u(\xi)$ and its derivatives with respect to $\xi$.

Step 2: We assume the solution of Eq.(15) can be revealed by a polynomial in $\phi(\xi)$ and $\psi(\xi)$ as follows:

$$
u(\xi)=\sum_{i=0}^{N} a_{i} \phi^{i}+\sum_{i=1}^{N} b_{i} \phi^{i-1} \psi
$$

where $a_{i}, b_{i}, c, \lambda, \mu$ are unknown constants to be determined later and by using homogeneous balance method we find out the value of positive integer $N$.

Step 3: Substituting the value of $N$ into (16) and inserting the modified equation of (16) into (15), using (6) and (8) (for case 1), the left-hand side of (15) can be moved into a polynomial of $\phi$ and $\psi$ wherein the degree of $\psi$ is not greater than one and the degree of $\phi$ is from zero to any positive integer. Equating like powers of the polynomial to zero, we attain a system of algebraic equations in $a_{i}, b_{i}, c, \lambda(\lambda<0), \mu, A_{1}$ and $A_{2}$.

Step 4: Using the algebraic equations in the Step 3 with the aid of Mathematica we get the values of $a_{i}, b_{i}, c, \lambda(\lambda<0)$, $\mu, A_{1}$ and $A_{2}$. Substituting this arbitrary values into the changed equation of (16) after putting the value of $N$, we can obtain the traveling wave solutions expressed by the hyperbolic functions and finally we get the solution of the nonlinear partial differential equation (15) i. e. (13) after substituting the coordinate transformation (14).

Step 5: Similar to step 3 and step 4, substitute Eq. (16) into Eq. (15) along with (6) and (10) for $\lambda>0$ (or Eq. (6) and Eq. (12) for $\lambda=0$ ) we obtain the traveling wave solutions of equation (15) i.e. equation (13) expressed by trigonometric functions (or by rational functions) respectively.

\section{Formulation of wave solutions}

In order to investigate new and further general wave solutions to the Burgers, Bogoyavlenskii and Negative Gardner-KP Equations in this section, we make use the two variable $\left(G^{\prime} / G, 1 / G\right)$-expansion method.

\subsection{Exact solutions of the Burgers equation}

In this subsection, we examine enormous fresh wave solutions to the Burgers equation (1) through the $\left(G^{\prime} / G, 1 / G\right)$ expansion method described in Section 2. For the Burgers equation, we use the coordinate transformation given in Eq. (14) to reduce Eq. (1) into the following ODE:

$$
-c u+1 / 2 u^{2}-u^{\prime}+k_{1}=0
$$

where $k_{1}$ is the integration constant and the prime denotes the derivation with respect to $\xi$. By balancing the Eq. (17) yields the balance number $N=1$. From Section 2, we attain that the solution of Eq. (17) has the following form

$$
u(\xi)=a_{0}+a_{1} \phi(\xi)+b_{1} \psi(\xi)
$$

where $a_{0}, a_{1}$ and $b_{1}$ are constants to be determined below. There are three cases to be discussed for solving the NLEE (1), as given in Section 2.

Case 1: When $\lambda<0$ (hyperbolic function solution),

Substituting (18) into (17) and using (6) and (8), the left-hand side of Eq. (17) becomes a polynomial in $\phi(\xi)$ and $\psi(\xi)$. 
Taking the coefficient of this polynomial to zero, gives a system of algebraic equations in $a_{0}, a_{1}, b_{1}, \mu, \lambda k_{1}$ and solving these algebraic equations by using Mathematica, we obtain two sets of values of constants as follows:

(i) $a_{0}=c, a_{1}=-1, b_{1}= \pm \frac{\sqrt{-\mu^{2}-\lambda^{2} \sigma}}{\sqrt{\lambda}}, k_{1}=\frac{\lambda+c^{2}}{2}$

(ii) $a_{0}=c, a_{1}=-2, b_{1}=0, \mu=0, k_{1}=\frac{4 \lambda+c^{2}}{2}$

Now for the above values of constants, the subsequent solutions of the equation (17) are obtained:

(i) Substituting the values of constants from (19)into solution (18), we obtain

$$
u(\xi)=c-\phi(\xi) \pm \frac{\sqrt{-\mu^{2}-\lambda^{2} \sigma}}{\sqrt{\lambda}} \psi(\xi)
$$

Using(5) and (7) into solution Eq. (21), we attain the solution of (17) as follows:

$$
\begin{aligned}
u(\xi) & =c-\frac{\sqrt{-\lambda}\left(A_{1} \cosh (\sqrt{-\lambda} \xi)+A_{2} \sinh (\sqrt{-\lambda} \xi)\right)}{A_{1} \sinh (\sqrt{-\lambda} \xi)+A_{2} \cosh (\sqrt{-\lambda} \xi)+\frac{\mu}{\lambda}} \\
& \pm \frac{\sqrt{-\mu^{2}-\lambda^{2} \sigma}}{\sqrt{\lambda}\left(A_{1} \sinh (\sqrt{-\lambda} \xi)+A_{2} \cosh (\sqrt{-\lambda} \xi)+\frac{\mu}{\lambda}\right)}
\end{aligned}
$$

Substituting the wave variable $\xi=x-c t$ into the above equation yields the solution of (1) as follows:

$$
\begin{aligned}
u(x, t) & =c-\frac{\sqrt{-\lambda}\left(A_{1} \cosh (\sqrt{-l}(x-c t))+A_{2} \sinh (\sqrt{-\lambda}(x-c t))\right)}{A_{1} \sinh (\sqrt{-\lambda}(x-c t))+A_{2} \cosh (\sqrt{-\lambda}(x-c t))+\frac{\mu}{\lambda}} \\
& \pm \frac{\sqrt{-\mu^{2}-\lambda^{2} \sigma}}{\sqrt{\lambda}\left(A_{1} \sinh (\sqrt{-\lambda}(x-c t))+A_{2} \cosh (\sqrt{-\lambda}(x-c t))+\frac{\mu}{\lambda}\right)}
\end{aligned}
$$

wherein $A_{1}$ and $A_{2}$ are arbitrary constants. Therefore, if we consider $A_{1}=0, A_{2} \neq 0$ and $\mu=0$ in (22), the solitary wave solution turns to be,

$$
u(x, t)=c-\sqrt{-\lambda} \tanh (\sqrt{-\lambda}(x-c t)) \pm \frac{\sqrt{-\lambda \sigma}}{A_{2}} \operatorname{sech}(\sqrt{-\lambda}(x-c t))
$$

On the other hand, if we consider $A_{1} \neq 0, A_{2}=0$ and $\mu=0$ in (22), the solitary wave solution becomes,

$$
u(x, t)=c-\sqrt{-\lambda} \operatorname{coth}(\sqrt{-\lambda}(x-c t)) \pm \frac{\sqrt{-\lambda \sigma}}{A_{1}} \operatorname{cosech}(\sqrt{-\lambda}(x-c t))
$$

(ii) Now, taking into consideration the values given in Eq. (20), solution Eq. (18), gives

$$
u(\xi)=c-2 \phi(\xi)
$$

where $\xi=x-c t$. Inserting (5) and (7) into Eq. (25), the solution of (1.1) converted into

$$
u(x, t)=c-\frac{2 \sqrt{-\lambda}\left(A_{1} \cosh (\sqrt{-\lambda}(x-c t))+A_{2} \sinh (\sqrt{-\lambda}(x-c t))\right)}{\left(A_{1} \sinh (\sqrt{-\lambda}(x-c t))+A_{2} \cosh (\sqrt{-\lambda}(x-c t))\right)}
$$


Since $A_{1}$ and $A_{2}$ are integral constant, in particular, if we put $A_{1}=0$ and $A_{2} \neq 0$ in (26), the solution turns into the kink shape soliton

$$
u(x, t)=c-2 \sqrt{-\lambda} \tanh (\sqrt{-\lambda}(x-c t))
$$

On the other hand, if we put $A_{1} \neq 0$ and $A_{2}=0$ into (26), the solution turns into the singular kink shape soliton

$$
u(x, t)=c-2 \sqrt{-\lambda} \operatorname{coth}(\sqrt{-\lambda}(x-c t))
$$

Case 2: When $\lambda>0$ (trigonometric function solutions), we executing the similar step as discussed in Case 1 . In this case, we obtain an over determine set of algebraic equations (for minimalism the equations are omitted here) and solving these equations the unknown constants are obtained as follows:

$$
\begin{aligned}
& \text { (i) } a_{0}=c, a_{1}=-1, b_{1}= \pm \frac{\sqrt{\lambda^{2} \rho-\mu^{2}}}{\sqrt{\lambda}}, k_{1}=\frac{\lambda+c^{2}}{2} \\
& \text { (ii) } a_{0}=c, a_{1}=-2, b_{1}=0, \mu=0, k_{1}=\frac{4 \lambda+c^{2}}{2}
\end{aligned}
$$

For the above sets of values of constants, we obtain the following solutions of the Eq. (1). (i) By means of values of (29), from (18), we obtain

$$
u(\xi)=c-\phi(\xi) \pm \frac{\sqrt{\lambda^{2} \rho-\mu^{2}}}{\sqrt{\lambda}} \psi(\xi)
$$

where $\xi=x-c t$. Substituting (9) into (5) and using this in Eq. (31), the following general solitary wave solution of (1) is obtained

$$
\begin{aligned}
u(x, t) & =c-\frac{\sqrt{\lambda}\left(A_{1} \cos (\sqrt{\lambda}(x-c t))-A_{2} \sin (\sqrt{\lambda}(x-c t))\right)}{A_{1} \sin (\sqrt{\lambda}(x-c t))+A_{2} \cos (\sqrt{\lambda}(x-c t))+\frac{\mu}{\lambda}} \\
& \pm \frac{\sqrt{\lambda^{2} \rho-\mu^{2}}}{\sqrt{\lambda}\left(A_{1} \sin (\sqrt{\lambda}(x-c t))+A_{2} \cos (\sqrt{\lambda}(x-c t))+\frac{\mu}{\lambda}\right)}
\end{aligned}
$$

(ii) Inserting the values from Eq. (30) into Eq. (18), we ascertain

$$
u(\xi)=c-2 \phi(\xi)
$$

wherein $\xi=x-c t$. Setting (5) and (9) into equation (33), the solution of (1) extracted as

$$
u(x, t)=c-\frac{2 \sqrt{\lambda}\left(A_{1} \cos (\sqrt{\lambda}(x-c t))-A_{2} \sin (\sqrt{\lambda}(x-c t))\right)}{A_{1} \sin (\sqrt{\lambda}(x-c t))+A_{2} \cos (\sqrt{\lambda}(x-c t))}
$$

Case 3: When $\lambda=0$ (rational function solution), Executing the parallel course of algorithm as Case 1, after solving the system of equations, we derive the values of constants as:

$$
\begin{aligned}
& \text { (i) } a_{0}=c, a_{1}=-1, b_{1}= \pm \sqrt{A_{1}^{2}-2 \mu A_{2}}, k_{1}=\frac{c^{2}}{2} \\
& \text { (ii) } a_{0}=c, a_{1}=-2, b_{1}=0, \mu=0, k_{1}=\frac{c^{2}}{2}
\end{aligned}
$$

Furthermore, for these two sets of values of constants, we attain the following solutions of the equation (1): 
(i) By means of the values of constants from Eq. (35) into Eq. (18), we achieve

$$
u(\xi)=c-\phi(\xi) \pm \sqrt{A_{1}^{2}-2 \mu A_{2}} \psi(\xi)
$$

where $\xi=x-c t$. Applying (5) and (11) into Eq. (37), the solution of (1) is obtained as follows

$$
\begin{array}{r}
u(x, t)=c-\frac{\mu(x-c t)+A_{1}}{\frac{\mu}{2}(x-c t)^{2}+A_{1}(x-c t)+A_{2}} \\
\quad \pm \frac{\sqrt{A_{1}^{2}-2 \mu A_{2}}}{\frac{\mu}{2}(x-c t)^{2}+A_{1}(x-c t)+A_{2}}
\end{array}
$$

(ii) Substituting the values of constants from (36) to Eq. (18)

$$
u(\xi)=c-2 \phi(\xi)
$$

Applying (5) and (11) into Eq. (39), the solution of (1) as follows:

$$
u(x, t)=c-\frac{2 A_{1}}{A_{1}(x-c t)+A_{2}}
$$

The solutions of Eq. (1) given in Eq. (32), Eq. (34) and Eq.(39) are new and further general than the solutions existing in the literature. If we choose further values of the arbitrary constants, different closed form solutions to the Burgers equation can be extracted, but for straightforwardness and conciseness the remaining solutions have not been sketched.

\subsection{Exact solutions of the Bogoyavlenskii equation}

In this subsection, by means of the suggested method, we extract traveling wave solutions as well as soliton solutions to the nonlinear evolution equation (2). The coordinate transformation for this case is as follows:

$$
u(x, y, t)=u(\xi), \quad v(x, y, t)=v(\xi), \quad \xi=x+y-c t
$$

where $c$ is the velocity of the traveling wave. By means of the above transformation the Bogoyavlenskii equation (2) will be turned into an ODE as follows:

$$
4 c u^{\prime}+u^{\prime \prime \prime}-4 u^{2} u^{\prime}-4 u^{\prime} v=0
$$

and

$$
u u^{\prime}=v^{\prime}
$$

Integrating (42) with respect to $\xi$ and omitting integrating constant, we obtain

$$
u^{2}=2 v
$$

Inserting Eq.(43) into Eq. (41) and integrating with respect to $\xi$ and setting the constant of integration equal to zero, yields

$$
u^{\prime \prime}-2 u^{3}+4 c u=0
$$


By balancing the highest order derivative term $u^{\prime \prime}$ and the nonlinear term $u^{3}$ appearing in Eq. (44), gives the balance number $N=1$. Thus, the solution of Eq. (44) becomes

$$
u(\xi)=a_{0}+a_{1} \phi(\xi)+b_{1} \psi(\xi)
$$

where $a_{0}, a_{1}$ and $b_{1}$ are constants to be calculated afterwards. There are three cases to be discussed which is introduced in section 2 .

Case 1: When $\lambda<0$ (Hyperbolic function solution), examining the analogous steps as subsection 3.1, it yields a system of algebraic equations and analyzing these equations give three sets of values of the constants as follows:

(i) $a_{0}=0, a_{1}= \pm \frac{1}{2}, b_{1}= \pm \frac{\sqrt{-\mu^{2}-\lambda^{2} \sigma}}{2 \sqrt{\lambda}}, c=\frac{-\lambda}{8}$
(ii) $a_{0}=0, a_{1}= \pm 1, b_{1}=0, c=\frac{-\lambda}{2}, \mu=0$
(iii) $a_{0}=0, a_{1}=0, b_{1}= \pm \sqrt{-\lambda \sigma}, c=\frac{\lambda}{4}, \mu=0$

For the above sets of the values of the constants, we find the subsequent solutions of the Bogoyavlenskii Eq. (2):

(i) Inserting (46) into Eq.(45) yields

$$
u(\xi)= \pm \frac{1}{2} \phi(\xi) \pm \frac{\sqrt{-\mu^{2}-\lambda^{2} \sigma}}{2 \sqrt{\lambda}} \psi(\xi)
$$

By means of (5) and (7), solution (49) turns into

$$
\begin{aligned}
u(\xi)= & \pm \frac{\sqrt{-\lambda}\left(A_{1} \cosh (\sqrt{-\lambda} \xi)+A_{2} \sinh (\sqrt{-\lambda} \xi)\right)}{2\left(A_{1} \sinh (\sqrt{-\lambda} \xi)+A_{2} \cosh (\sqrt{-\lambda} \xi)+\frac{\mu}{\lambda}\right)} \\
& \pm \frac{\sqrt{-\mu^{2}-\lambda^{2} \sigma}}{2 \sqrt{\lambda}\left(A_{1} \sinh (\sqrt{-\lambda} \xi)+A_{2} \cosh (\sqrt{-\lambda} \xi)+\frac{\mu}{\lambda}\right)}
\end{aligned}
$$

Substituting the value of into (43), we attain

$$
\begin{aligned}
v(\xi)=\left[\frac{\sqrt{-\lambda}\left(A_{1} \cosh (\sqrt{-\lambda} \xi)+A_{2} \sinh (\sqrt{-\lambda} \xi)\right)}{2\left(A_{1} \sinh (\sqrt{-\lambda} \xi)+A_{2} \cosh (\sqrt{-\lambda} \xi)+\frac{\mu}{\lambda}\right)}\right. \\
\left.\quad+\frac{\sqrt{-\mu^{2}-\lambda^{2} \sigma}}{2 \sqrt{\lambda}\left(A_{1} \sinh (\sqrt{-\lambda} \xi)+A_{2} \cosh (\sqrt{-\lambda} \xi)+\frac{\mu}{\lambda}\right)}\right]^{2},
\end{aligned}
$$

where $\xi=x+y+\frac{\lambda t}{8}$. The solutions (50) and (51) are the solitary wave solutions to the (2+1)-dimensional Bogoyavlenskii equation.

(ii) For the values of the parameters accumulated in (47) yields the succeeding solution of the Bogoyavlenskii equation (2):

$$
u(\xi)= \pm \frac{\sqrt{-\lambda}\left(A_{1} \cosh (\sqrt{-\lambda} \xi)+A_{2} \sinh (\sqrt{-\lambda} \xi)\right)}{A_{1} \sinh (\sqrt{-\lambda} \xi)+A_{2} \cosh (\sqrt{-\lambda} \xi)}
$$

and

$$
v(\xi)=\frac{1}{2}\left[\frac{\sqrt{-\lambda}\left(A_{1} \cosh (\sqrt{-\lambda} \xi)+A_{2} \sinh (\sqrt{-\lambda} \xi)\right)}{A_{1} \sinh (\sqrt{-\lambda} \xi)+A_{2} \cosh (\sqrt{-\lambda} \xi)}\right]^{2}
$$


wherein $\xi=x+y+\frac{\lambda t}{2}$.

(iii) For the values of the parameters gathered in (48) gives the subsequent solution of Eq. (2):

$$
u(\xi)= \pm \frac{\sqrt{-\lambda \sigma}}{A_{1} \sinh (\sqrt{-\lambda} \xi)+A_{2} \cosh (\sqrt{-\lambda} \xi)}
$$

and

$$
v(\xi)=\frac{1}{2}\left[\frac{\sqrt{-\lambda \sigma}}{A_{1} \sinh (\sqrt{-\lambda} \xi)+A_{2} \cosh (\sqrt{-\lambda} \xi)}\right]^{2}
$$

wherein $\xi=x+y-\frac{\lambda t}{4}$. Solutions (50)-(55) are generalized solitary wave solutions to the (2+1)-dimensional Bogoyavlenskii equation. Setting definite values of the associated parameters diverse known soliton solutions can be determined. But, for conciseness the special solutions are not scored here.

Case 2: When $\lambda>0$ (trigonometric function solution), executing the similar steps like subsection 3.1, it produces a system of algebraic equations and analyzing these equations give subsequent values of the parameters:
(i) $a_{0}=0, a_{1}= \pm \frac{1}{2}, b_{1}= \pm \frac{\sqrt{-\mu^{2}+\lambda^{2} \rho}}{2 \sqrt{\lambda}}, c=\frac{-\lambda}{8}$
(ii) $a_{0}=0, a_{1}= \pm 1, b_{1}=0, c=\frac{-\lambda}{2}, \mu=0$
(iii) $a_{0}=0, a_{1}=0, b_{1}= \pm \sqrt{\lambda \rho}, c=\frac{\lambda}{4}, \mu=0$

For the above values of the constants, we attain the following solutions of the equation (2):

(i) Inserting the values of the parameters provided in (56) into the solution Eq. (45),

$$
u(\xi)= \pm \frac{1}{2} \phi(\xi) \pm \frac{\sqrt{-\mu^{2}+\lambda^{2} \rho}}{2 \sqrt{\lambda}} \psi(\xi)
$$

By means of (5) and (9), from (59), we obtain the following general solitary wave solution of (2)

$$
\begin{aligned}
u(\xi)= & \pm \frac{\sqrt{\lambda}\left(A_{1} \cos (\sqrt{\lambda} \xi)-A_{2} \sin (\sqrt{\lambda} \xi)\right)}{2\left(A_{1} \sin (\sqrt{\lambda} \xi)+A_{2} \cos (\sqrt{\lambda} \xi)+\frac{\mu}{\lambda}\right)} \\
& \pm \frac{\sqrt{-\mu^{2}+\lambda^{2} \rho}}{2 \sqrt{\lambda}\left(A_{1} \sin (\sqrt{\lambda} \xi)+A_{2} \cos (\sqrt{\lambda} \xi)+\frac{\mu}{\lambda}\right)}
\end{aligned}
$$

and

$$
\begin{aligned}
v(\xi)=\left[\frac{1 \sqrt{\lambda}\left(A_{1} \cos (\sqrt{\lambda} \xi)-A_{2} \sin (\sqrt{\lambda} \xi)\right)}{2\left(A_{1} \sin (\sqrt{\lambda} \xi)+A_{2} \cos (\sqrt{\lambda} \xi)+\frac{\mu}{\lambda}\right)}\right. \\
\left.\quad+\frac{\sqrt{-\mu^{2}+\lambda^{2} \rho}}{2 \sqrt{\lambda}\left(A_{1} \sin (\sqrt{\lambda} \xi)+A_{2} \cos (\sqrt{\lambda} \xi)+\frac{\mu}{\lambda}\right)}\right]^{2}
\end{aligned}
$$

where $\xi=x+y+\frac{\lambda t}{8}$. 
(ii) For values of the constants given in (57), computing similar gradations we extract the solution of Eq. (2) as,

$$
u(\xi)= \pm \frac{\sqrt{\lambda}\left(A_{1} \cos (\sqrt{\lambda} \xi)-A_{2} \sin (\sqrt{\lambda} \xi)\right)}{A_{1} \sin (\sqrt{\lambda} \xi)+A_{2} \cos (\sqrt{\lambda} \xi)}
$$

and

$$
v(\xi)=\frac{1}{2}\left[\frac{\sqrt{\lambda}\left(A_{1} \cos (\sqrt{\lambda} \xi)-A_{2} \sin (\sqrt{\lambda} \xi)\right)}{A_{1} \sin (\sqrt{\lambda} \xi)+A_{2} \cos (\sqrt{\lambda} \xi)}\right]^{2}
$$

wherein $\xi=x+y+\frac{\lambda t}{2}$.

(iii) Again for the constants provided in (58), we obtain the solutions of (2) as,

$$
u(\xi)= \pm \frac{\sqrt{\lambda \rho}}{A_{1} \sin (\sqrt{\lambda} \xi)+A_{2} \cos (\sqrt{\lambda} \xi)}
$$

and

$$
v(\xi)=\frac{1}{2}\left[\frac{\sqrt{\lambda \rho}}{A_{1} \sin (\sqrt{\lambda} \xi)+A_{2} \cos (\sqrt{\lambda} \xi)}\right]^{2}
$$

wherein $\xi=x+y-\frac{\lambda t}{4}$.

Case 3: When $\lambda=0$ (rational function solution), implementing the analogous steps as subsection 3.1, it offers a cluster of algebraic equations and solving this system of equations give following values of the parameters:

(i) $a_{0}=0, a_{1}= \pm \frac{1}{2}, b_{1}= \pm \frac{1}{2} \sqrt{A_{1}^{2}-2 \mu A_{2}}, c=0$

(ii) $a_{0}=0, a_{1}=0, b_{1}= \pm A_{1}, \mu=0, c=0$

Furthermore for the above values of constants, we have the following solutions of the equation (2):

(i) For (66) from (45), we obtain

$$
u(\xi)= \pm \frac{1}{2} \phi(\xi) \pm \frac{\sqrt{A_{1}^{2}-2 \mu A_{2}}}{2} \psi(\xi)
$$

By means of (5) and (11) from (48), we obtain

$$
u(\xi)= \pm \frac{\mu \xi+A_{1}}{2\left(\frac{\mu}{2} \xi^{2}+A_{1} \xi+A_{2}\right)} \pm \frac{\sqrt{A_{1}^{2}-2 \mu A_{2}}}{2\left(\frac{\mu}{2} \xi^{2}+A_{1} \xi+A_{2}\right)}
$$

and

$$
v(\xi)=\frac{1}{2}\left[\frac{\mu \xi+A_{1}}{2\left(\frac{\mu}{2} \xi^{2}+A_{1} \xi+A_{2}\right)} \frac{\sqrt{A_{1}^{2}-2 \mu A_{2}}}{2\left(\frac{\mu}{2} \xi^{2}+A_{1} \xi+A_{2}\right)}\right]^{2}
$$

where $\xi=x+y$.

(ii) For (67) from (45) and using (5) and (11) the solution turns out into

$$
u(\xi)= \pm \frac{A_{1}}{A_{1} \xi+A_{2}}
$$


and

$$
u(\xi)=\frac{1}{2}\left[\frac{A_{1}}{A_{1} \xi+A_{2}}\right]^{2}
$$

If we set special values of $A_{1}$ and $A_{2}$ into the solutions gained from case 2 and 3 , it can be found further known solutions consistently through this method, but for minimalism and brevity the remaining solutions have not been written down.

\subsection{Exact solutions of the negative Gardner-KP equation}

In the current section, we examine abundant explicit close form wave solutions to the negative Gardner-KP equation (3). For this equation, we make use of the subsequent coordinate transformation:

$$
u(x, y, t)=u(\xi), \quad \xi=k(x+y-v t)
$$

where $k$ and $v$ are constant to be determine afterword.

Using this wave transformation Eq. (3) converts into an ODE and integrating twice, we obtain

$$
(1-v) u+3 u^{2}-2 u^{3}+k^{2} u^{\prime \prime}+c=0
$$

where $c$ is the integrating constant and the first integrating constant is considered to zero. The balance between the highest order derivative term $u^{\prime \prime}$ and the nonlinear term $u^{3}$ appearing in Eq. (73), yields the balance number $N=1$. So, the solution of Eq. (73) has the form

$$
u(\xi)=a_{0}+a_{1} \phi(\xi)+b_{1} \psi(\xi)
$$

where $a_{0}, a_{1}$ and $b_{1}$ are constants to be evaluated later. Depending on the attribute of $\lambda$ there will arise three incidents:

Case 1: When $\lambda<0$, putting through the steps as subsection 3.1 and solving the system of algebraic equations yield three sets of values of the constants as follows:

(i) $a_{0}=\frac{1}{2}, a_{1}= \pm \frac{k}{2}, b_{1}= \pm \frac{i k \sqrt{\mu^{2}+\lambda^{2} \sigma}}{2 \sqrt{\lambda}}, v=\frac{5+\lambda k^{2}}{2}, c=\frac{1+\lambda k^{2}}{4}$

(ii) $a_{0}=\frac{1}{2}, a_{1}= \pm k, b_{1}=0, v=\frac{5+4 \lambda k^{2}}{2}, c=\frac{1+4 \lambda k^{2}}{4}, \mu=0$

(iii) $a_{0}=\frac{1}{2}, a_{1}=0, b_{1}= \pm i k \sqrt{\lambda \sigma}, v=\frac{5-2 \lambda k^{2}}{2} c=\frac{1-2 \lambda k^{2}}{4}, \mu=0$

For the above sets of values, we attain the following solutions of the Eq. (73):

(i) By means of (75), from (74) it yields

$$
u(\xi)=\frac{1}{2} \pm \frac{k}{2} \phi(\xi) \pm \frac{i k \sqrt{\mu^{2}+\lambda^{2} \sigma}}{2 \sqrt{\lambda}} \psi(\xi)
$$

Making use of (5) and (7) into Eq. (78), we attain the solution of (3) as,

$$
\begin{aligned}
u(\xi)= & \frac{1}{2} \pm \frac{k \sqrt{-\lambda}\left(A_{1} \cosh (\sqrt{-\lambda} \xi)+A_{2} \sinh (\sqrt{-\lambda} \xi)\right)}{2\left(A_{1} \sinh (\sqrt{-\lambda} \xi)+A_{2} \cosh (\sqrt{-\lambda} \xi)+\frac{\mu}{\lambda}\right)} \\
& \pm \frac{i k \sqrt{\mu^{2}+\lambda^{2} \sigma}}{2 \sqrt{\lambda}\left(A_{1} \sinh (\sqrt{-\lambda} \xi)+A_{2} \cosh (\sqrt{-\lambda} \xi)+\frac{\mu}{\lambda}\right)}
\end{aligned}
$$


where $\xi=x+y-\frac{5+\lambda k^{2}}{2}, \sigma=A_{1}^{2}-A_{2}^{2}$ and $\mu$ is an arbitrary constant.

(ii) Substituting (76) into Eq. (74), we attain

$$
u(\xi)=\frac{1}{2} \pm k \phi(\xi)
$$

Inserting (5) and (7) into Eq. (80), the following general solitary wave solution of Eq. (3) is obtained

$$
u(\xi)=\frac{1}{2} \pm \frac{k \sqrt{-\lambda}\left(A_{1} \cosh (\sqrt{-\lambda} \xi)+A_{2} \sinh (\sqrt{-\lambda} \xi)\right)}{A_{1} \sinh (\sqrt{-\lambda} \xi)+A_{2} \cosh (\sqrt{-\lambda} \xi)}
$$

where $\xi=x+y-\frac{5+4 \lambda k^{2}}{2} t$.

(iii) Utilizing (77) into (74) and using Eq. (5) and Eq. (7), yields the solution of Eq. (3) as follows:

$$
u(\xi)=\frac{1}{2} \pm \frac{i k \sqrt{\lambda \sigma}}{A_{1} \sinh (\sqrt{-\lambda} \xi)+A_{2} \cosh (\sqrt{-\lambda} \xi)}
$$

wherein $\xi=x+y-\frac{5-2 \lambda k^{2}}{2} t$ and $\sigma=A_{1}^{2}-A_{2}^{2}$.

Case 2: When $\lambda>0$, carrying out similar process, we get three types of value of the constants as follows:
(i) $a_{0}=\frac{1}{2}, a_{1}= \pm \frac{k}{2}, b_{1}= \pm \frac{k \sqrt{-\mu^{2}+\lambda^{2} \rho}}{2 \sqrt{\lambda}}, v=\frac{5+\lambda k^{2}}{2}, c=\frac{1+\lambda k^{2}}{4}$
(ii) $a_{0}=\frac{1}{2}, a_{1}= \pm k, b_{1}=0, v=\frac{5+4 \lambda k^{2}}{2}, c=\frac{1+4 \lambda k^{2}}{4}, \mu=0$
(iii) $a_{0}=\frac{1}{2}, a_{1}=0, b_{1}= \pm i k \sqrt{\lambda \rho}, v=\frac{5-2 \lambda k^{2}}{2} c=\frac{1-2 \lambda k^{2}}{4}, \mu=0$

For the above sets of constants, we attain the following solutions of the equation (73):

(i) Using (83) into (74), yields

$$
u(\xi)=\frac{1}{2} \pm \frac{k}{2} \phi(\xi) \pm \frac{k \sqrt{-\mu^{2}+\lambda^{2} \rho}}{2 \sqrt{\lambda}} \psi(\xi)
$$

By means of (5) and (7) solution (86) the solution turns out into,

$$
\begin{aligned}
u(\xi)=\frac{1}{2} & \pm \frac{k \sqrt{\lambda}\left(A_{1} \cos (\sqrt{\lambda} \xi)-A_{2} \sin (\sqrt{\lambda} \xi)\right)}{2\left(A_{1} \sin (\sqrt{\lambda} \xi)+A_{2} \cos (\sqrt{\lambda} \xi)+\frac{\mu}{\lambda}\right)} \\
& \pm \frac{k \sqrt{-\mu^{2}+\lambda^{2} \rho}}{2 \sqrt{\lambda}\left(A_{1} \sin (\sqrt{\lambda} \xi)+A_{2} \cos (\sqrt{\lambda} \xi)+\frac{\mu}{\lambda}\right)}
\end{aligned}
$$

where $\xi=x+y-\frac{5+\lambda k^{2}}{2} t, \rho=A_{1}^{2}+A_{2}^{2}$ and $\mu$ is an arbitrary constants.

(ii) Substituting (84) into (74) and by means of (5) and (7), we attain the following closed form solution of Eq. (3):

$$
u(\xi)=\frac{1}{2} \pm \frac{k \sqrt{\lambda}\left(A_{1} \cos (\sqrt{\lambda} \xi)-A_{2} \sin (\sqrt{\lambda} \xi)\right)}{A_{1} \sin (\sqrt{\lambda} \xi)+A_{2} \cos (\sqrt{\lambda} \xi)}
$$


where $\xi=x+y-\frac{5+4 \lambda k^{2}}{2} t$.

(iii) By means of (85) the solution becomes,

$$
u(\xi)=\frac{1}{2} \pm \frac{k \sqrt{\lambda \rho}}{A_{1} \sin (\sqrt{\lambda} \xi)+A_{2} \cos (\sqrt{\lambda} \xi)}
$$

wherein $\xi=x+y-\frac{5-2 \lambda k^{2}}{2} t$ and $\rho=A_{1}^{2}+A_{2}^{2}$.

Case 3: When $\lambda=0$, we obtain three types of value of constants as follows:

(i) $a_{0}=\frac{1}{2}, a_{1}= \pm \frac{k}{2}, b_{1}= \pm \frac{k \sqrt{A_{1}^{2}-2 \mu A_{2}}}{2}, v=\frac{5}{2}, c=\frac{1}{4}$

(ii) $a_{0}=-\frac{1}{2}, a_{1}=0, b_{1}= \pm k A_{1}, v=\frac{5}{2}, c=\frac{1}{4}, \mu=0$

Furthermore for the above values of constants we have the following solutions of the equation (3):

(i) Using (90) into solution Eq. (74), gives

$$
u(\xi)=\frac{1}{2} \pm \frac{k}{2} \phi(\xi) \pm \frac{k \sqrt{A_{1}^{2}-2 \mu A_{2}}}{2} \psi(\xi)
$$

By means of (5) and (11) from Eq. (93), the solution of Eq.(3) becomes

$u(\xi)=\frac{1}{2} \pm \frac{k\left(\mu \xi+A_{1}\right)}{2\left(\frac{\mu}{2} \xi^{2}+A_{1} \xi+A_{2}\right)} \pm \frac{k \sqrt{A_{1}^{2}-2 \mu A_{2}}}{2\left(\frac{\mu}{2} \xi^{2}+A_{1} \xi+A_{2}\right)}$

where $\xi=x+y-\frac{5}{2} t$.

(ii) Employing (91), (74) and using the conditions for rational function solution gives the solution of Eq. (3) as follows:

$$
u(\xi)=\frac{1}{2} \pm \frac{k A_{1}}{A_{1} \xi+A_{2}}
$$

where $\xi=x+y-\frac{5}{2} t$.

As $A_{1}$ and $A_{2}$ are arbitrary constants one might randomly select their values. Therefore, selecting random values, we might found further traveling wave solutions of (3) but we omitted them for simplicity. Remark: All solutions have been checked with Mathematica by putting them into the original equation (1), (2) and (3) and found correct.

\section{Results and discussion}

In this article, the two variable $\left(G^{\prime} / G, 1 / G\right)$-expansion method has successfully been implemented to investigate abundant wave solutions of the (1+1)-dimensional Burgers equation, the (2+1)-dimensional Bogoyavlenskii equation and the (2+1)dimensional negative Gardner-KP equation. We have obtained three types of solutions, such as hyperbolic, trigonometric and rational function. The two variable $\left(G^{\prime} / G, 1 / G\right)$-expansion method turns into the $\left(G^{\prime} / G\right)$-expansion method if we set $\mu=0$ into Eq. (4) and $b_{i}=0$ into Eq. (16). It is noteworthy to observe that abundant exact solutions of the considered equations have been developed. The attained wave solutions represent different known solitons, as for instance bell shape 
soliton, kink soliton, singular kink soliton, periodic soliton, singular periodic soliton etc. These types of solutions are very much important to analyze the associated physical phenomena. The shape of the solutions (3.1.24) and (3.1.11) are identical to (15) and (16) respectively obtained in ref. [35], since the geometrical meaning of a solution is independent of arbitrary constants. For the Bogoyavlenskii equation, the shape of the solutions (71) and (72)are similar to (33) and (39) derived in [36]. Also, the solution (3.3.9) is similar to the solution of the equation attained in [37]. The rest of the solutions, we attain in this article are new and have not been achieved in the previous literature. By using this important method, we have determined huge amount of closed form wave solutions of the considered equations which provides much information to understand intricate physical phenomena.

\section{Conclusion}

In this article, we established further general and some new exact traveling wave solutions to the $(1+1)$-dimensional Burgers equation, the $(2+1)$-dimensional Bogoyavlenskii equation and the (2+1)-dimensional negative Gardner-KP equation through the two variable $\left(G^{\prime} / G, 1 / G\right)$-expansion method. We assert that the solutions obtained in this article can be used in signal processing system, optical fiber, a distinct topological signature in the phase space etc. The solutions may be worthy to use in turbulent flow, theory of shock wave, stochastic process, complex solitary wave solution, solitary waves in shallow water and in many other places. If the parameters receive special values, the prominent solitary wave solutions, like bell shape soliton, kink, singular soliton, periodic solitons etc. are established. The most important advantage of this method over the other methods is that, it possesses all the three types of solutions as hyperbolic function, trigonometric function and rational function. This study shows that the two variable $\left(G^{\prime} / G, 1 / G\right)$-expansion method is quite efficient and practically well suited to find exact traveling wave solutions for the problems considered in this article and similar to the considered problems.

\section{Competing interests}

The authors declare that they have no competing interests.

\section{Authors' contributions}

All authors have contributed to all parts of the article. All authors read and approved the final manuscript.

\section{References}

[1] B.R. Parkes, E.J.and Duffy. An automated tanh-function method for finding solitary wave solutions to nonlinear evolution equations. Comput. Phys. Commun., 98:288-300, 1996.

[2] S.K. Liu, Z.T. Fu, S.D. Liu, and Q. Zhao. Jacobi elliptic function expansion method and periodic wave solutions of nonlinear wave equation. Physics Letter A, 289:69-74, 2001.

[3] Z. Fu, S. Liu, and Q. Zhao. New jacobi elliptic function expansion and new periodic solutions of nonlinear wave equations. Physics Letter A, 290:72-76, 2001.

[4] M. L. Wang. Solitary wave solutions for variant boussinesq equations. Physics Letter A, 199, 1995.

[5] E. Fan and H. Zhang. A note on the homogeneous balance method. Physics Letter A, 246:403-406, 1998.

[6] M.L. Wang and Y.B. Zhou. The periodic wave solutions for the klein-gordon-schrodinger equation. Physics Letter A, 318:84-92, 2003. 
[7] M.L. Wang, X.Z. Li, and J.L. Zhang. The sub-ode method and solitary wave solutions for higher order nonlinear schrodinger equation. Cogent Mathematics, 363:96-101, 2007.

[8] J.H. He and X.H. Wu. Exp-function method for nonlinear wave equations. Chaos, Solitons and Fractals, 30, 2006.

[9] J.H. He and M.A. Abdou. New periodic solutions for nonlinear evolution equations using exp-function method. Chaos, Solitons and Fractals, 34, 2007.

[10] J.H. He X.H. Wu. Exp- function method and its application to nonlinear equations. Chaos, Solitons and Fractals, 38:903-910, 2008.

[11] Z.Z. Ganji, D.D. Ganji, and H. Bararnia. Approximate general and explicit solution of nonlinear bbmb equations by exp-function method. Applied Mathematics and Modeling, 30:1836-1841, 2009.

[12] Y. Huang. New no-traveling wave solutions for the liouville equation by backlund transformation method. Nonlinear Dynamics, 72:87-90, 2013.

[13] M. Wadati, H. Sanuki, and K. Konno. Relationships among inverse method, backlund transformation and an infinite number of conservation laws. Progress in Theoritical Physics, 53:419-436, 1975.

[14] W Malfliet. Solitary wave solutions of nonlinear wave equations. Americal Journal of Physics, 60:650-654, 1992.

[15] W. Malfliet and W. Hereman. The tanh method: I. exact solutions of nonlinear evolution and wave equations. Physica Scripta, 54:563-568, 1996.

[16] D.D. Ganji and M. Abdollahzadeh. Exact travelling solutions for the lax's seventh-order kdv equation by sech method and rational exp-function method. Applied Mathematics and Computation, 206:438-444, 2008.

[17] E. Fan. Extended tanh-function method and its applications to nonlinear equations. Physics Letter A, 277:212-218, 2000.

[18] A.M. Wazwaz. The sine-cosine method for obtaining solutions with compact and non-compact structures. Applied Mathematics and Computation, 159:559-576, 2004.

[19] A.M. Wazwaz and M.A. Helal. Nonlinear variants of the bbm equation with compact and non-compact physical structures. Chaos, Solitons and Fractals, 26:767-776, 2006.

[20] A.M. Wazwaz. A sine-cosine method for handling nonlinear wave equations. Mathematics, Computation and Modlling, 40:499-508, 2004.

[21] L.X. Li and M. L. Wang. The $\left(\mathrm{G}^{\prime} / \mathrm{G}\right)$-expansion method and travelling wave solutions for a higher-order nonlinear schrodinger equation. Applied Mathematics and Computation, 208:440-445, 2009.

[22] M.L. Wang, X.Z. Li, and J.L. Zhang. The $\left(\mathrm{G}^{\prime} / \mathrm{G}\right)$-expansion method and travelling wave solutions of nonlinear evolution equations in mathematical physics. Physics Letter A, 372:417-423, 2008.

[23] M.A. Akbar and N.H.M. Ali. Exp-function method for duffing equation and new solutions of (2+1)-dimensional dispersive long wave equations. Progress in Applied Mathematics, 1(2):30-42, 2011.

[24] M.A. Akbar, N.H.M. Ali, and E.M.E. Zayed. Abundant exact traveling wave solutions of the generalized bretherton equation via ( $\left.\mathrm{G}^{\prime} / \mathrm{G}\right)$-expansion method. Communication in Theoritical Physics, 57(2):173-178, 2012.

[25] M.A. Akbar, N.H.M. Ali, and E.M.E. Zayed. A generalized and improved $\left(\mathrm{G}^{\prime} / \mathrm{G}\right)$-expansion method for nonlinear evolution equations. Math. Prob. Engr., 2012:22, 2012.

[26] E.M.E. Zayed and S. Al-Joudi. Application of an extended $\left(\mathrm{G}^{\prime} / \mathrm{G}\right)$-expansion method to find exact solutions of nonlinear pdes in mathematical physics. Applied Mathematics and Computation, 2010:19, 2010.

[27] L. X. Li, E. Q. Li, and M. L. Wang. The $\left(\mathrm{G}^{\prime} / \mathrm{G}, 1 / \mathrm{G}\right)$-expansion method and its application to traveling wave solutions of the zakharov equations. Applied Mathematics-A Journal of Chinese Universities, 25(4):454-462, 2010.

[28] E. M. E. Zayed and M. A. M. Abdelaziz. The two variables $\left(\mathrm{G}^{\prime} / \mathrm{G}, 1 / \mathrm{G}\right)$-expansion method for solving the nonlinear kdv-mkdv equation. Mathematical Problem in Engineering, 2012:725061, 2012.

[29] E. M. E. Zayed, S. A. H. Ibrahim, and M. A. M. Abdelaziz. Traveling wave solutions of the nonlinear (3+1)dimensional kadomtsev- petviashvili equation using the two variable $\left(\mathrm{G}^{\prime} / \mathrm{G}, 1 / \mathrm{G}\right)$-expansion method. Journal of Applied Mathematics, 2012:560531, 2012. 
[30] S. Demiray, O. Unsal, and A Bekir. New exact solutions for boussinesq type equations by using $\left(\mathrm{G}^{\prime} / \mathrm{G}, 1 / \mathrm{G}\right)$ and (1/G)-expansion methods. ACTA Physics Polonica A, 125(5), 2014.

[31] S. Demiray, O. Unsal, and A Bekir. Exact solutions of nonlinear wave equations using $\left(\mathrm{G}^{\prime} / \mathrm{G}, 1 / \mathrm{G}\right)$-expansion method. Journal of the Egyptian Mathematical Society, 23(1):78-84, 2015.

[32] M. M. Miah, H. M. S. Ali, and M. A. Akbar. An investigation of abundant traveling wave solutions of complex nonlinear evolution equations: The perturbed nonlinear schrodinger equation and the cubic-quintic ginzburg-landau equation. Cogent Mathematics, 3:1277506, 2016.

[33] H. M. S. Ali, M. M. Miah, and M. A. Akbar. Study of abundant explicit wave solutions of the drinfeld-sokolovsatsuma-hirota (dssh) equation and the shallow water wave equation. Propulsion and Power Research, 7(4):320-328, 2018.

[34] M. M. Miah, H. M. S. Ali, M. A. Akbar, and A. M. Wazwaz. Some applications of the $\left(\mathrm{G}^{\prime} / \mathrm{G}, 1 / \mathrm{G}\right)$-expansion method to find new exact solutions of nlees. The European Physical Journal Plus, 132(252), 2017.

[35] H. Kheiri, M.R. Moghaddam, and V. Vafaei. Application of the $\mathrm{G}^{\prime} / \mathrm{G}$-expansion method for the burgers, burgershuxley and modified burgers-kdv equations. Pramana Journal of Physics, 76(6):833-842, 2011.

[36] S. Xie, S. Heo, S. Kim, G. Woo, and S. Yi. Numerical solution of one-dimensional burgers equation using reproducing kernel function. Journal of Computational and Applied Mathematics, 214:417-434, 2008.

[37] I.and Kumar H. Malik, A.and Chand and S.C. Mishra. Exact solutions of the bogoyavlenskii equation using the multiple G' /G-expansion method. Computational Mathematics and Applied, 64:2850-2859, 2012.

[38] Y. Peng and M. Shen. On exact solution of the bogoyavlenskii equation. Pramana Journal of Physics, 67(3):447-456, 2006.

[39] Z. Shafiof, S.M.and Bagheri and A. Sousaraei. New solutions for positive and negative gardner-kp equation. World Appl. Sci. J., 13(4):662-666, 2011.

[40] X. Bin and L. X. Qiang. Classification, reduction, group invariant solutions and conservation laws of the gardner-kp equations. Applied Mathematics and Computation, 215:1244-1250, 2009. 